\title{
Una nueva relación del Tercer sector y la economía social
}

\author{
New relation between Third Sector and social economy
}

\author{
Teresa CRESPO JULIA \\ Consell assessor de Politiques Socials \\ i familiars de la Generalitat de Catalunya \\ teresa.crespo@ono.com
}

Recibido: $27 / 11 / 2012$

Revisado: 04/12/2012

Aceptado: 21/03/2013

Disponible on line: 16/04/2013

\section{Resumen}

Este artículo plantea algunos puntos del debate acerca de la naturaleza del Tercer Sector como sector no lucrativo, y su identificación o no con el concepto de economía social. La consideración relevante es que se trata de formas de economía, diferentes a las mercantiles, que son desconocidas o poco conocidas en la sociedad. La función de la Ley de Economía social española busca una mejor definición de las entidades que la componen, así como fomentar su desarrollo y su visibilidad. Sin embargo todavía existen resistencias, a pesar de las cuales el Tercer sector ha sabido profesionalizarse y organizarse en los últimos años, convirtiéndose en un sector con potencialidad económica que ha mejorado extraordinariamente su gestión y su capacidad de respuesta eficaz y de calidad. Los cambios que están obligados a realizar en la forma de gestión, tanto el Tercer Sector como la Economía social en el periodo de crisis actual, no van a alterar en estas entidades los valores de solidaridad, inclusión social, utilidad pública y cohesión social y territorial, porque forman parte de la naturaleza y los principios de estas entidades. No hay que olvidar que el Tercer sector tiene entre sus objetivos principales conseguir oportunidades que permitan la inserción laboral de aquellas personas que tienen dificultades para trabajar. La economía social se contempla como una vía de inserción activa de las personas en riesgo de exclusión y con déficits personales para incorporarse al mercado laboral ordinario y ejercer sus derechos de ciudadanía.

Palabras clave: Tercer Sector social, economía social, impacto social, valores sociales, inclusión social, cohesión social, economía solidaria.

\begin{abstract}
This paper poses some of the points of discussion about the Third Sector nature as a non profit sector, and its identification or not with the concept of social economy. Relevant consideration is that they are forms of economy, different from the market economy, unknown or little known by the society. The Social Economy Spanish Law seeks a better definition of the entities related as well as promoting their development and visibility. Notwithstanding, there remain some resistances in spite of which the Third Sector has known how to professionalize and organize itself along the last years, becoming a sector of economic potential having improved enormously its management and its ability to give a quality and efficient response. Changes in the forms of management due to be made by the Third Sector and the Social Economy in these times of crisis, are not going to alter these entities values of solidarity, social inclusion, public usefulness and social and territorial cohesion, because they constitute part of their nature and principles. One should not forget that the Third Sector has among its main goals that of providing opportunities allowing the social inclusion of those with difficulties to work. Social Economy is regarded as a way to the active insertion of those persons at risk of exclusion and with handicaps to join the normal labour market and exercise their citizen rights.
\end{abstract}

Keywords: Third social sector, social economy, social impact, social values, social inclusion, social cohesion, solidarity economy.

Referencia normalizada: Crespo Julia, T. (2013): «Una nueva relación del Tercer sector y la economía social». Cuadernos de Trabajo Social, 26(1): 65-74.

Sumario: Introducción. 1. El Tercer sector social y la economía social dos componentes de una misma realidad. 2. Trazos específicos del Tercer sector. 3. Razones del Tercer sector en defensa de la economía social. 4. Estrategias del Tercer sector para fortalecer la economía social. 5. Referencias bibliográficas. 


\section{Introducción}

En estos momentos de crisis profunda en que todo se cuestiona y nada nos sirve como antes, estamos obligados a modificar nuestra manera de intervenir, de relacionarnos, de atender a las personas, de gestionar y financiar nuestros proyectos, y evidentemente también es imprescindible modificar nuestra mirada respecto a la economía. En este panorama un tanto inseguro, la economía social, que históricamente ha jugado un papel secundario en nuestra sociedad, está llamada a modificar su función, su representación y su impacto.

1. El Tercer sector social y la economía social dos componentes de una misma realidad Intentaré dar algunas pinceladas sobre qué es uno y otro concepto, para después dibujar algunas vías que permiten articular y potenciar a ambos en una misma realidad. Al Tercer sector social y a la economía social les unen unas características y objetivos comunes. Ambas son organizaciones privadas que centran su actividad en trabajar para mejorar la sociedad y atender las necesidades de la persona y no las del capital; aplican criterios de gestión para optimizar su actividad y no para conseguir un mayor margen económico; buscan el beneficio de la comunidad y no el propio; focalizan su actuación en incrementar la rentabilidad y aprovechamiento de los recursos y no en dominar el mercado y priorizan los resultados sociales y no los económicos, que si los tuvieren los reinvierten en la propia organización (Argudo López, 2002).

La economía social tiene sus orígenes en las cooperativas de consumo y producción y en las mutualidades de finales del siglo XVIII y principios del siglo XIX, defienden una economía diferente a la predominante, en la que unen la actividad económica a los valores y a la ética, adoptando como bandera la honestidad, la transparencia y la responsabilidad social, y cuestionando las verdades que el capitalismo siempre ha defendido, como son la libertad de mercado como único elemento regulador, y el interés económico particular como motor de la economía. Entre los modelos referentes de este sector económico encontramos las cooperativas, como el mayor exponente pero no el único de la economía social, según Monzón (citado en Argudo López, pp. 12-16). El cooperativismo se ha reafirmado como columna vertebral de la econo- mía social y con los años este sector ha ido evolucionado y definiéndose con mayor claridad. Será la Comisión de la Comunidad Europea quien en su Consejo en 1989 declaraba a:

Las cooperativas, asociaciones y mutuas como las organizaciones de economía social que aplican técnicas de organización especificas a una actividad productiva. Estas técnicas se basan en los principios de solidaridad y participación de los miembros, productores, usuarios o consumidores, y en valores como la autonomía y la ciudadanía.

Dichas organizaciones se han desarrollado, pero con un crecimiento desigual y nunca han tenido la suficiente fuerza para enfrentarse al sistema capitalista, que ha determinado la economía del mundo occidental. Quizás, actualmente han definido más claramente su espacio y desde él cuestionan con mayor fuerza el modelo económico dominante que actúa bajo los intereses del mercado y en beneficio de una pequeña minoría, en un mundo globalizado donde las desigualdades son cada día mayores.

Algunos sectores han puesto en cuestión si verdaderamente el sector no lucrativo es parte integrante de dicha economía, y a ello ha contribuido la falta de homogeneidad en su composición, su clasificación y su nomenclatura. En países como Francia o España entendemos la economía social en sentido amplio, y por lo tanto, incluimos en ella al Tercer sector social; mientras otros países como Gran Bretaña o Alemania defienden una opción más restrictiva y consideran el principio lucrativo como una razón fundamental para separar las ONGs del mundo de la economía social.

Existe pues una falta de claridad de criterios en su catalogación. Algunos intentos, como los realizados por la Universidad norteamericana de Johns Hopkins han ayudado a su clarificación al considerar que una institución puede ser considerada del Tercer sector social si cumple los siguientes criterios:

a) Organización formal con objetivos, estructura y estatuto legal.

b) Privada, no formando parte del sector público, aunque éste pueda ser uno de los principales recursos económicos.

c) No lucrativa, que significa que no se reparten beneficios, que se destinan a los fines de la entidad. 
d) Autogobierno de la propia entidad, disponiendo de una regulación propia.

e) Participación voluntaria y donaciones altruistas para el desarrollo de sus actividades que junto al trabajo remunerado llevan a cabo sus objetivos (p. 11).

Finalmente señalaría que los últimos estudios realizados y la propia ley española de economía social optan claramente por el criterio de que el hecho de ser o no una entidad lucrativa no es un factor determinante ni excluyente para formar parte de la economía social, y en cambio, se hace hincapié en la no maximización de los beneficios económicos, que se considera la característica más específica del mundo capitalista.

Otras voces dicen que la característica que da la especificidad a la economía social, es la manera de tomar las decisiones. Defienden que éstas han de tomarse de forma participativa y ha de existir una clara relación con las personas receptoras del servicio, ayuda o prestación, es decir con los beneficiarios, condición que determina la función social de la entidad (Pérez Giner, citado en Argudo López, p. 25). Aquí de nuevo figuran la cooperativa como la organización por excelencia de la economía social, seguida de otras como las asociaciones, las mutuas, las sociedades laborales y diferentes agrupaciones de productores. En un sentido amplio se contemplan las fundaciones que no se caracterizan por tener un sistema de autogestión, pero sí por sus objetivos sociales, las empresas de inserción, los centros especiales de empleo, y por último encontramos las empresas públicas de interés general, como la ONCE que se la cataloga como organización singular en el marco de la economía social. Estas organizaciones, como hemos ya apuntado, pueden ser más o menos diversas y es un conjunto de modelos organizacionales no homogéneo, en la que se dan una diversidad de actores y una variedad de actividades y gestión, pero que a pesar de sus diferencias podemos identificar unas características comunes que las enmarca y las define en el si de dicho concepto.

Con la aprobación de la Ley 5/2011 de Economía social se consigue clarificar este conjunto un tanto diverso, y definir un marco jurídico común para la diversidad de entidades que actúan en la economía social, identificarlas para darles visibilidad, potenciarlas y fomentarlas. Una aportación importante de esta normativa, es la definición de los principios orientadores de la ley que especifica las características comunes del conjunto de entidades que se mueven con criterios y objetivos sociales y gestionan de forma empresarial para conseguir unos buenos resultados que las hagan sostenibles. La ley destaca como trazos principales:

a) Primacía de las personas y el fin social sobre el capital, que se concreta en la gestión autónoma y transparente, democrática y participativa que lleva a priorizar la toma de decisiones más en función del fin social, que en relación a sus aportaciones al capital social.

b) Aplicación de los resultados obtenidos de la actividad económica principalmente en función del trabajo aportado y servicio o actividad realizada por las socias u socios o por sus miembros y en su caso, al fin social de la entidad.

c) Promoción de la solidaridad interna y con la sociedad que favorezca el compromiso con el desarrollo local. La igualdad de oportunidades entre hombres y mujeres, la cohesión social, la inserción de personas en riesgo de exclusión social, la generación de empleo estable y de calidad, la conciliación de la vida personal, familiar y laboral y la sostenibilidad.

d) Independencia respecto a los poderes públicos (art. 4).

Esta ley ha permitido poner un poco de orden en la clasificación de las distintas tipologías proponiendo recogerlas en un catálogo específico. Después de haber definido la peculiaridad de sus valores y su configuración específica, también ha facilitado la incorporación de nuevos modelos de organizaciones, aunque todavía falta completar la identificación de nuevas iniciativas del Tercer sector que son nuevos inputs en la economía social. A la vista de lo que hemos comentado, está bastante claro que cuando hablamos de este concepto no se trata únicamente de las cooperativas, las SALT, las mutualidades, las fundaciones, las asociaciones, las empresas de inserción, etc., sino que debemos pensar en otras alternativas que permiten su consolidación, como son las cláusulas sociales, y sus dispositivos, los talleres ocupacionales, los centros especiales de trabajo entre otros, como recursos del Tercer sector muy adecuados para la inserción y la creación de ocupación para colectivos vulnerables, y también como nuevas opciones que fortalecen a la economía social. 
Quisiera destacar que restan por aplicar aspectos que la ley apuntaba para potenciar y desarrollar dichas organizaciones. En ese sentido, las políticas actuales tienen una tarea pendiente que permita dar a les entidades el protagonismo que les corresponde, y en especial, destacaría la débil implantación de aquellas medidas de fomento y difusión que en el art. 8 las concreta en: «remover los obstáculos que impidan su desarrollo», o "crear un entorno que fomente su desarrollo» o «involucar a las entidades de la economía social en las políticas activas de empleo, especialmente a favor de los sectores más afectados por el desempleo, mujeres, jóvenes y parados de larga duración».

Lamentablemente, todavía la economía social y más aún, las entidades que contribuyen a su desarrollo no han conseguido el reconocimiento de la sociedad, y en muchas ocasiones son las grandes desconocidas, porque no se ha hecho el esfuerzo de valorar política ni socialmente su aportación social y económica. Tenemos ante nosotros el reto de identificar y darles el necesario apoyo a las nuevas maneras existentes para conseguir que esta economía diferente se incremente y sea una verdadera alternativa para la ocupación de los colectivos que más lo necesitan. Existe pues, un camino trazado gracias a la ley, pero resta todavía un gran trecho por recorrer para su total desarrollo y aplicación.

\section{Trazos específicos del Tercer sector}

A continuación trataremos del Tercer sector social con el fin de comprender mejor qué significa en el contexto actual. Tiene su origen en aquellas iniciativas privadas de carácter caritativo y religioso de antaño, y paulatinamente ha ido incorporando nuevos perfiles altruistas defensores de la justicia social que han asumido el nuevo protagonismo de la sociedad civil que se siente corresponsable junto a los poderes públicos, del bienestar de la comunidad. Esta evolución del sector ha permitido mejorar su organización y estructuración y a su vez ha introducido técnicas y métodos de gestión consiguiendo una mayor sostenibilidad de sus proyectos, sin abandonar los fines que motivaron su creación, como es trabajar en defensa de la cohesión y justicia social. En definitiva, dicho sector que durante tiempo lo definíamos poco profesionalizado, atomizado y con débiles es- tructuras, se ha ido articulando y ha sabido en los últimos años organizarse, convirtiéndose en un sector con potencialidad económica, mejorando extraordinariamente su gestión y su capacidad de respuesta eficaz y de calidad.

En el marco del Tercer sector social debemos destacar el valor de la aportación voluntaria. España no es tradicionalmente un país con una fuerte presencia de organizaciones o movimientos de voluntarios, y en los últimos años en parte de la mano de las entidades sociales ha ido creciendo y se ha ido organizando. Ha experimentado una mejora cualitativa muy positiva, ha sido capaz de definir sus funciones y roles, ha regulado su actuación, ha fijado claramente sus responsabilidades, competencias, formación, y derechos y deberes para el ejercicio de sus actividades, siendo hoy un elemento determinante en la mayoría de las organizaciones sin ánimo de lucro, en las que se contemplan como un valor añadido que complementa la acción social de los distintos profesionales de las entidades. El voluntariado forma parte del sector, es un valor intrínseco del mismo, y se encuentra integrado en la mayoría de las entidades.

El sector social se ha desarrollado gracias a la actividad de las organizaciones sociales y la iniciativa privada que se articula y se manifiesta de muy distintas maneras con formas diversas para conseguir un mayor bienestar entre la población, siempre actuando bajo los principios que le han caracterizado, como son la solidaridad, no tener ánimo de lucro, y la lucha contra las desigualdades. Su trayectoria en los últimos años nos muestra una clara complicidad entre lo público y lo privado, gracias a la actuación de la Administración pública o primer sector que en tiempos de bonanza económica optó por externalizar muchos de sus servicios y confió su gestión a las entidades sin ánimo de lucro, ello ha hecho que este sector se haya caracterizado por trabajar en buena parte en la producción de bienes y servicios de interés público, siendo uno de los brazos ejecutores de la administración, y ocupando un lugar importante en el conjunto de la economía del país.

Pero este crecimiento que últimamente ha experimentado el sector lleva implícito ese pecado original que tarde o temprano le pasa factura, y es la excesiva dependencia de las enti- 
dades sociales de los recursos públicos. El sector se ha convertido en un gestor y prestador de servicios públicos, que en ocasiones lo ha conseguido licitando abiertamente la contratación del sector público, pero otras veces ha aceptado la gestión de dichos servicios por la vía de la subvención que a la larga genera un fuerte debilitamiento de la sostenibilidad de los proyectos, de la capacidad económica y del poder de decisión de las entidades.

Esta práctica, que durante años ha generado un fuerte crecimiento del sector, actualmente debido a les restricciones de los presupuestos públicos, está teniendo una influencia en sentido contrario, y está ayudando al proceso de reducción de su actividad y los recursos. Junto a este cambio con respecto a la Administración nos encontramos con otros elementos que dificultan también su desarrollo: nos referimos a que las organizaciones sociales deben convivir con las empresas mercantiles que con una clara voluntad de obtener máximos beneficios en interés propio entran en competencia con las entidades sociales en un contexto dominado por la economía financiera y los intereses de la banca, que les genera mayores dificultades para vencer los diversos frentes adversos a su crecimiento y sostenibilidad.

Este panorama, que en ciertos ámbitos está alcanzando visos de gravedad, nos muestra el nuevo protagonismo de la economía social y solidaria que aparece como una pequeña rendija por donde salvarse, a la vez que se valora como una vía alternativa para potenciar y dar viabilidad a las entidades sociales, y por ser además un elemento fundamental para luchar contra el desempleo. Por ello, el sector está apostando por empresas productivas no lucrativas con la colaboración entre lo público y lo privado, olvidando la dependencia única y exclusiva anterior, potenciando la inversión privada procedente de una mayor corresponsabilidad de la sociedad civil, y planificando también nuevas maneras de financiarse y de gestionar la actividad que realizan.

Estos cambios en la gestión económica no obvian la defensa de los principios de siempre, y con estos valores impulsa toda iniciativa, individual o colectiva, de autoempleo o pequeños grupos que tengan algún proyecto y quieran iniciar una actividad, consiguiendo, en parte y a pesar de las muchas dificultades, superar el cambio de la coyuntura actual, en el que cada día con mayor fuerza, la administración deja de ser su principal fuente de financiación.

Este proceso ha ido dando mayor valor económico a la actividad que desarrolla el sector, y ello ha hecho que exista cada vez más una clara identificación con la economía social, creciendo y desarrollándose uno al lado de la otra, y de alguna manera, ambas formen parte de un mismo concepto del que han sido causa y efecto de su reciente evolución, que de forma tímida se está definiendo como una economía que pasa por lo social y quiere ser una alternativa al capitalismo. Y así nos lo muestran los datos sobre la economía social de 2012 facilitados por la Confederación empresarial CEPES, que nos dicen que genera aproximadamente el 7,5 por ciento del PIB, con más de 43.000 empresas, más de 2.390 .000 empleos, más de 12.500.000 de personas asociadas y una facturación de 76.000.000.000 euros.

\section{Razones del Tercer sector en defensa de la economía social}

En este apartado intentaré identificar las razones que nos inducen a afirmar que el Tercer sector social es una línea fuerza de la economía social. Como referencia, señalaría el estudio realizado por la Taula del Tercer sector social de Catalunya, titulado «El valor añadido del tercer sector en la prestación de los servicios públicos. Guía práctica de cláusulas sociales en la contratación pública de Servicios Sociales y de atención a las personas (2009) y el realizado por CEPES, titulado «El impacto socioeconómico de las entidades de Economía social» (2011) profundizan en esta materia. A continuación enumeramos algunos de los argumentos que genera el sector:

- La utilidad social. En el estudio realizado por CEPES considera que dicho concepto tiene un carácter multidimensional compuesto por los siguientes elementos: unos criterios económicos y sociales que permiten obtener un servicio o producto a menor coste, gracias al ahorro que significa la aportación de la mano de obra voluntaria, que en el ultimo Anuario del Tercer sector de Acción social en España (2010) nos mostraba representa un 62,3 por ciento del personal que trabaja en el conjunto de entidades; igualmente la actividad 
de muchas de sus organizaciones tiene como objeto potenciar la igualdad de oportunidades y trabajar en favor de las personas más débiles para conseguir su inserción social y laboral; valora la proximidad y la creación de vínculos sociales; resalta la innovación social, económica e institucional; y por último, destaca la utilidad interna que genera una mejora en la manera de funcionar la organización permitiendo la participación. De la síntesis de estos criterios se define la utilidad pública, entendida como el resultado de la actividad encaminada a contribuir en la reducción de las desigualdades económicas y sociales.

- La mejora de la cohesión social y territorial. Se entiende por una sociedad cohesionada, aquella que la ciudadanía tiene un sentido de pertenencia a una comunidad, participa como sujeto de derechos y deberes, y goza de una mínima redistribución equitativa de los recursos. En consecuencia, siempre que se trabaje por construir una sociedad más justa y menos exclusiva se conseguirá mayor cohesión entre la población, y en la medida que genera unos recursos económicos que se distribuyen según los criterios de justicia redistributiva de las entidades sociales, se beneficia a un mayor número de población, como es el personal laboral, el voluntariado, y a todas las personas que reciben atención o ayuda.

Un defecto que en ocasiones se ha atribuido al Tercer sector es su dispersión en el territorio, pero esta característica aparentemente negativa puede convertirse en una cualidad, dependiendo de cómo se gestione, porque significa una mayor capilaridad en su ámbito de actuación, y por lo tanto, la mejor adecuación de unos servicios al territorio que gracias a su proximidad con las personas de una zona determinada es capaz de adaptarse y ser rápido en la respuesta adecuada. Esta realidad a su vez significa que si es viable su actividad, permite disponer de una red de servicios territorializados que posibilita una mayor cohesión social y una actividad económica que directa o indirectamente ayuda al desarrollo local.

- La rentabilidad social, concepto de difícil mesura económica, pero precisamente es uno de los elementos que más claramente define uno de los factores básicos de la economía social. Dicha rentabilidad se valora a través de los efectos de todas aquellas actividades que realizan las entidades sociales con objeto de ayudar, prestar un servicio, proporcionar un soporte, una guía, un consejo, una información, o una formación a la persona a fin de proporcionarle un mayor bienestar, una mayor autonomía, y en definitiva, conseguir su desarrollo integral que le permita actuar responsablemente como ciudadana y sujeto de derechos y deberes.

- Ser motor de ocupación. El Tercer Sector tiene como uno de los objetivos principales conseguir oportunidades que permitan la inserción laboral de aquellas personas que tienen dificultades para trabajar. La economía social se contempla como una vía de inserción activa en el que la persona en riesgo de exclusión y con déficits personales para incorporarse en el mercado laboral ordinario se ve obligada a ser sujeto activo como trabajador que ejerce su derecho al trabajo y debe esforzarse según sus competencias y posibilidades en producir bienes, y mejorar sus capacidades. Esta visión de las políticas activas de ocupación nos muestra una clara articulación entre los objetivos sociales ligados a la inserción laboral y aquellos que se refieren a la creación de actividad laboral de producción y generación de riqueza. Por otro lado, no se ha de olvidar que algunas de las personas que trabajan en este sector, no podrían ocuparse en otro tipo de empresas, y por lo tanto ello significa que se está facilitando contrataciones a colectivos que tienen alguna discapacidad o dificultad de insertarse laboralmente. El trabajo de dichas personas no siempre consigue el nivel de productividad que sería necesario para obtener una producción competitiva y sostenible, y en consecuencia estas entidades asumen de alguna u otra manera el déficit que se pueda producir, entendiendo que su actividad genera otros beneficios sociales que justifican este tipo de proyectos. La suma de estos dos factores, social y económico, tiene sin duda un retorno o impacto en la sociedad de un considerable valor y que nunca deberíamos obviar.

Si hemos hablado de la capacidad del sector en insertar a población en riesgo de exclusión, es además un nuevo yacimiento de ocupación gracias a que hasta hace muy poco ha ido incrementando la contratación de profesio- 
nales especializados en este sector, centrándose en actividades de atención a las personas y en la gestión de servicios sociales. Así, en el ámbito de la dependencia existe un alto nivel de demanda de servicios para las personas mayores y discapacitadas, como también en los servicios sociales y los servicios educativos. A pesar de esta evolución cabe recordar que la ley de la autonomía personal y de la dependencia se vio como uno de los nuevos motores para la creación de nuevos lugares de trabajo, y a posteriori, debido al débil desarrollo de la misma no se han podido alcanzar las previsiones que se hicieron. Con todo podemos afirmar que la dinámica positiva del mercado laboral de este sector ha producido una disminución de la tasa de paro entre sus profesionales.

Cabe considerar también, la importancia que ha ido adquiriendo los planes formativos en el marco de las políticas activas de ocupación, reciclajes, formación permanente, o formación ocupacional, etc. El conjunto de esta actividad representa un porcentaje elevado de profesionales que trabajan en esta tarea y significa también un volumen importante económicamente hablando, al que debemos añadir otras actuaciones relacionadas con la inserción sociolaboral como es la orientación o la mediación en las empresas que mueven un volumen importante de técnicos y de presupuestos públicos.

- La práctica de una política de recursos humanos diferente. A pesar de que no se ha conseguido en el sector social una política de recursos humanos que sea plenamente igualitaria, como constatamos en un estudio realizado por la Asociación de Entidades Catalanas de Acción Social (ECAS) sobre la conciliación en el sector, y también en datos del Anuario del sector social en Cataluña (2011), podemos apuntar que, siendo muy mayoritaria la presencia femenina en este tipo de entidades, existen menores diferencias por razón de género que en el mundo de la empresa ordinaria en temas como las carreras profesionales, niveles salariales o cuestiones relacionadas con la conciliación. Las mujeres de este sector junto al funcionariado son quienes disfrutan de mayor facilidad para conciliar el mundo laboral, familiar y privado. Sin embargo, todavía nos encontramos que en los puestos directivos están minoritariamente representadas las mujeres (30 por ciento), a pesar de que exista una mayoría de trabajadoras ( 70 por ciento de la plantilla). Estos datos que hacen referencia al Tercer Sector social no son del todo coincidentes con algunos estudios realizados sobre el conjunto de las entidades de economía social, donde se afirma que verdaderamente existe una equidad en las oportunidades entre hombre y mujer. Ello nos plantea la necesidad de que se debería analizar de nuevo esta cuestión, a fin de constatar si existen o no estas diferencias que hemos visto.

Cabe también destacar que el sector goza de una cierta calidad del empleo. Existe mayor estabilidad en los puestos de trabajo, ya que se tiene mayor consideración con los trabajadores, manteniéndose un dialogo más abierto y permanente con las personas contratadas. El sector habitualmente contempla el lugar de trabajo como un espacio de formación y de prácticas que permite a cada uno avanzar según sus posibilidades, y llevar a cabo un proceso de aprendizaje para poder participar plenamente en la actividad de la organización y progresar en la entidad.

Por último, constatamos que existe un mayor porcentaje de contratación temporal, ya que es habitual el contrato por obra, debido en parte al tipo de relación contractual que se mantiene con la Administración, que ha estipulado para financiar los servicios que externaliza, la modalidad de convenios de uno o dos años, o la vía de la subvención que conlleva casi siempre la obligatoriedad de presentarse a convocatorias anuales. Esta práctica significa una cierta inseguridad en la continuidad de los proyectos, dificultando la contratación indefinida, siendo un grave inconveniente para las entidades del tercer sector que se encuentran en la más absoluta indefensión para programar su actividad a largo término, y perjudicando directamente al personal contratado que no puede conseguir una cierta seguridad en su trabajo.

- Mejora la gestión de sus organizaciones. Como hemos apuntado el Tercer sector ha evolucionado muchísimo en temas relacionados con la gestión, y cada día es más profesional, al haber aplicado en su trabajo criterios técnicos y económicos de gestión y organiza- 
ción. Se ha profesionalizado, ha regularizado al personal técnico, lo ha formado, ha cuidado las relaciones laborales, ha planificado sus actividades y ha fijado objetivos sociales, pero también ha determinado los objetivos económicos sin olvidar criterios de cualidad. En síntesis, podríamos decir que las entidades del Tercer Sector se han transformado en organizaciones gestionadas con criterios empresariales sin abandonar los fines sociales que originaron su creación. Esa nueva manera de actuar, dirigir y gestionar las organizaciones sociales ha dado lugar, en buena parte, el crecimiento de la economía social, potenciando la especificidad social que ésta tiene.

- Su actividad y producción tiene menores costes económicos. Es evidente que la gestión de muchos de los servicios que el sector social produce representa un ahorro para la Administración responsable de los mismos. Las razones son: porque no se dan niveles salariales desorbitados, no se contemplan primas ni contratos blindados entre los directivos, por el contrario, existe una aportación importante de colaboraciones altruistas, de una base social que colabora, de un voluntariado que dedica su tiempo a ayudar a las personas y dar viabilidad a muchos proyectos que sin estas aportaciones difícilmente serian posibles. Otro aspecto a considerar es que son organizaciones que se definen por no tener ánimo de lucro, y por lo tanto los costes empresariales no contemplan el margen de beneficios, y en caso que hubiera alguna ganancia ésta se reinvierte en la propia entidad. Todo ello da como resultado unos costes inferiores al de las empresas mercantiles.

\section{Estrategias del Tercer sector para forta- lecer la economía social}

Apuntamos algunos aspectos que son o pueden ser verdaderos motores de la economía social, e intentaremos identificar algunos de estos factores diferenciales que el Tercer sector aporta al fortalecimiento de la economía social. Conocemos que la economía social se desarrolla en los márgenes de los núcleos de acumulación de capital, crece gracias a un tipo de organizaciones diferentes, donde existen nuevas maneras de gestionar, de actuar y de obtener resultados que solo pueden ser reconocidos dentro de los parámetros de dicha economía.
En momentos de crisis nos preguntamos si ésta nos proporciona nuevas oportunidades. Sinceramente creo que sí, y podemos constatar que en la actualidad están surgiendo otras formas de relacionarse, de trabajar y de intercambiar. Si habíamos conocido experiencias en Latinoamérica que se basaban en el trueque y el intercambio, e incluso en la creación de una moneda social, una unidad de cambio o de tiempo, sin ninguna relación con la moneda circulante, ahora nos encontramos en nuestro entorno con iniciativas minoritarias que recuerdan esa realidad que nos parecía muy lejana. Conocemos barrios o zonas delimitadas que han empezado de manera informal a intercambiar servicios, objetos, comida, o cualquier otra cosa que una persona puede ofrecer a cambio de algo que necesita, y partiendo de este modelo están surgiendo iniciativas sociales $\mathrm{y}$ redes informales que promueven bancos comunitarios de alimentos, de intercambio de tiempo, de experiencias, de conocimientos $\mathrm{y}$ saber Esta práctica nos lleva a un modelo socioeconómico diferente en el que no existe el lucro, no existe el interés privado, y únicamente se busca satisfacer aquellos mínimos que se precisan para vivir dignamente. No pretendemos defender el retorno a la economía de subsistencia o al trueque, pero sí que esos ejemplos han dado lugar a que los servicios sociales y algunas entidades sociales se hayan planteado ese modelo participativo y comunitario, como un recurso para potenciar y generar una mayor cohesión social. Como un ejemplo de nuevas experiencias podemos consultar $L e L a-$ bo de l'économie sociale et solidaire (ESS), una asociación francesa que impulsa identifica y coordina todas aquellas experiencias que pueden reconocerse como economía social respetuosa con la persona y su entorno (www.lelabo-ess.org.).

Jeremy Rifkin en su obra «El fin del trabajo» (2004), plantea el paso a una nueva etapa de la historia humana en el que el trabajo ya no existirá como hasta ahora, a la vista de que el número de los parados y los infra-empleados es cada día más elevado. Nos habla de la era post-mercado en la que se deberán pensar formas alternativas al trabajo tradicional, donde haya que poner en marcha nuevos maneras de disponer recursos y de repartirlos, y apunta que una mayor confianza en el Tercer sector 
debería permitir la reconstrucción de nuestras comunidades y nuestras culturas, dibujando formas como el trueque o intercambio muy relacionadas con las experiencias comentadas. Nos gustaría que estas vivencias en pequeña escala fueran el comienzo de una sociedad distinta y más equitativa en la que la economía social debería tener mucha más fuerza como alternativa que la actual economía de mercado.

Si cuando todavía no podíamos imaginar la profunda crisis que hoy estamos viviendo ya se hablaba de que se debía pensar en un cambio del concepto trabajo, ahora ya no nos queda ninguna duda que éste no va a ser posible para todo el mundo, y en consecuencia estamos obligados a repensar muchas de las cosas que hasta hoy han sido elementos que han sustentado nuestro estado del bienestar, como son: el reconocimiento del derecho al trabajo, las cotizaciones de los trabajadores que han permitido tener unas pensiones cuando se llega a la edad de jubilarnos y unas prestaciones en momentos de interrupción del trabajo por enfermedad o por desocupación; y en otro orden de cosas, tampoco podemos olvidar la regulación laboral que ha determinado el funcionamiento de buena parte de las relaciones sociales y económicas del país. Ante este nuevo escenario existe cada vez un mayor convencimiento de que la falta de ocupación nos obliga a ser capaces de buscar alternativas al trabajo, hasta hoy eje de nuestro modelo socioeconómico, y hemos de aprender a potenciar la capacidad de crear actividades que ayuden al desarrollo de la persona y de la comunidad y a mejorar así la cohesión social.

Sabemos que la ocupación es un concepto dinámico y variable según el proceso personal y el entorno social y territorial de cada uno, pero como nunca más se podrá responder plenamente a las demandas del mercado laboral, y por lo tanto, no podrá ser la vía para la integración de la ciudadanía. Debemos innovar en la búsqueda de nuevas vías de participación, de creación de redes, de espacios relacionales, de hacer y de actuar para el bienestar de la comunidad- En definitiva, estamos pensando en nuevas opciones para la integración de las personas en una sociedad que ha de ser más inclusiva y que, seguro, debe pasar por actividades que prioricen las personas y no el consumo ni el beneficio propio. En esta línea creemos que las experiencias que se están dando de empresariado comunitario o social son nuevos ejemplos de formas emergentes y todavía indefinidas, que nacen como diferentes maneras de subsistencia, pero que pueden llegar a ser alternativa a la producción del mercado en la que hasta ahora hemos estado viviendo.

Otro aspecto que el Tercer sector está trabajando prioritariamente es la estructuración de redes de colaboración y de comunicación que permitan llevar a cabo estrategias globales que canalicen esfuerzos y recursos en proyectos comunes, compartir la gestión y sistemas de financiación (banca ética), encontrar nuevas maneras de sumar potencialidades, saber prescindir del interés individual y buscar el beneficio de lo colectivo. Se pretende constituir una plataforma fuerte que nos ayude a darnos a conocer y que pueda ser una verdadera alternativa a los modelos hoy dominantes, es decir una mirada que nos lleva hacia una manera distinta de entender la economía.

Tampoco podemos olvidar la aportación a la nueva economía que representa la banca ética, los microcréditos o créditos solidarios que el premio Nóbel Muhammad Yunues inventó para financiar a personas humildes, como un instrumento para salir de la pobreza y luchar contra las leyes del mercado. Gracias a su aportación se ha practicado este modelo en otros lugares, alcanzando unos buenos resultados sociales y económicos, aunque todavía con escaso impacto en el conjunto del sector que hoy se encuentran sin posibilidad de encontrar financiación ni crédito para sus proyectos sociales.

A manera de conclusión, desde el Tercer sector social afirmamos que aportamos valor añadido en nuestra actividad, que se fundamenta en que éste es generador de valores, detecta nuevas necesidades sociales, instrumentaliza la existencia de vías de participación, incrementa la cohesión e inclusión social, implementa los servicios sociales de acuerdo con las necesidades, evalúa los servicios prestados, fomenta la iniciativa social, y sobre todo focaliza su acción en la persona, en responder a sus necesidades de la manera más rápida y adecuada. En definitiva, el Tercer sector desempeña un papel relevante en el desarrollo social en España y esa idea se consolida a través del incremento continuado del número de beneficia- 
rios directos, gracias a la acción de las entidades sociales, aunque todavía con escaso impacto en el conjunto del sector, que hoy se encuentra con una débil base social, y muchas dificultades para disponer de la financiación que necesita. Dicho valor añadido que acaba- mos de describir lo podemos identificar de manera muy parecida en la economía social, porque es parte integrante en ambas opciones que tienen como objetivo prioritario luchar contra las desigualdades y conseguir una sociedad más justa y equitativa.

\section{Referencias bibliográficas}

AA.VV. (2010). Anuario del tercer sector de la Acción social en España. Madrid: Fundación Luis Vives.

Argudo, J.L. (octubre, 2002). El tercer sector y la economía social. Revista Acciones e Investigaciones Sociales 15, 239-263.

Ayuntamiento de Barcelona. (2011). Quaderns de la xarxa sociolaboral 1 (XISL). Economia social i potencialitat de les xarxes. Barcelona: Ajuntament de Barcelona.

Confederación Empresarial Española de Economía Social. (septiembre 2011). El impacto socioeconómico de las entidades de economía social, identificación, medición y valoración de los efectos vinculados a los principios de actuación de las empresas de la economía. Madrid: CEPES.

García Delgado, J.L. (2009). Las Cuentas de la Economía Social. Magnitudes y la financiación del Tercer Sector en España. Madrid: Civitas-Thomson Reuters : Fundación ONCE.

Observatori del Tercer Sector y Taula d'entitats del Tercer Sector Social. ( 2011). Anuari del tercer sector social de Catalunya 2011. Barcelona: Observatori del Tercer Sector y Taula d'entitats del Tercer Sector Social.

Rifkin , J. (2004). El fin del trabajo. Barcelona: Paidós Ibérica.

Taula d'entitats del Tercer Sector social de Catalunya. (2009). El valor añadido del tercer sector en la prestación de los servicios públicos. Guía práctica de clausulas sociales en la contratación pública de servicios sociales y de atención a las personas. Barcelona: Taula d'entitats del Tercer Sector social de Catalunya.

Vidal, I. (2001). El libro blanco de la Economía social en Cataluña. Barcelona: Consejo de Economía Social e Investigación CIES. 